\title{
Anomalous spectral dependence of optical polarization and its impact on spin detection in InGaAs/GaAs quantum dots
}

Yuttapoom Puttisong, Yuqing Huang, Irina Buyanova, X. J. Yang, A. Subagyo, K. Sueoka, A. Murayama and Weimin Chen

\section{Linköping University Post Print}

\section{Tweet}

N.B.: When citing this work, cite the original article.

Original Publication:

Yuttapoom Puttisong, Yuqing Huang, Irina Buyanova, X. J. Yang, A. Subagyo, K. Sueoka, A. Murayama and Weimin Chen, Anomalous spectral dependence of optical polarization and its impact on spin detection in InGaAs/GaAs quantum dots, 2014, Applied Physics Letters, (105), 13, 132106.

http://dx.doi.org/10.1063/1.4897306

Copyright: American Institute of Physics (AIP) http://www.aip.org/

Postprint available at: Linköping University Electronic Press http://urn.kb.se/resolve?urn=urn:nbn:se:liu:diva-112185 


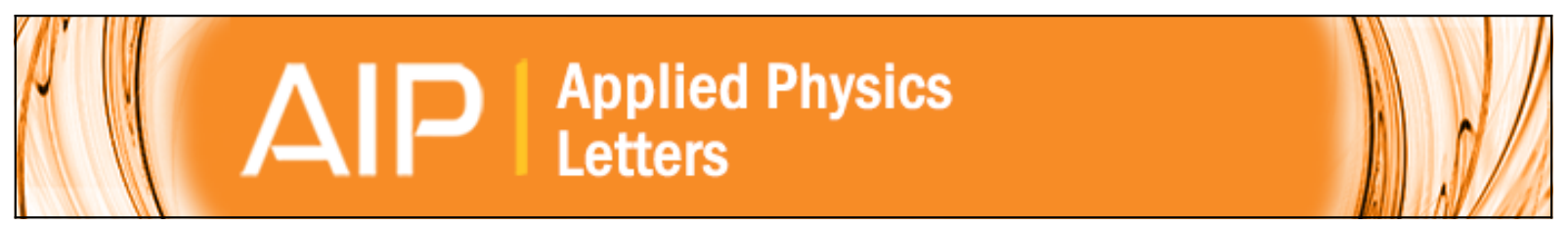

\section{Anomalous spectral dependence of optical polarization and its impact on spin detection in InGaAs/GaAs quantum dots}

Y. Puttisong, Y. Q. Huang, I. A. Buyanova, X. J. Yang, A. Subagyo, K. Sueoka, A. Murayama, and W. M. Chen

Citation: Applied Physics Letters 105, 132106 (2014); doi: 10.1063/1.4897306

View online: http://dx.doi.org/10.1063/1.4897306

View Table of Contents: http://scitation.aip.org/content/aip/journal/apl/105/13?ver=pdfcov

Published by the AIP Publishing

\section{Articles you may be interested in}

Growth-temperature dependence of optical spin-injection dynamics in self-assembled InGaAs quantum dots J. Appl. Phys. 116, 094309 (2014); 10.1063/1.4894712

Temperature dependence of the dynamics of optical spin injection in self-assembled InGaAs quantum dots Appl. Phys. Lett. 103, 082405 (2013); 10.1063/1.4819208

Strong room-temperature optical and spin polarization in InAs/GaAs quantum dot structures

Appl. Phys. Lett. 98, 203110 (2011); 10.1063/1.3592572

Optical spin-filtering effect in charged InAs/GaAs quantum dots

Appl. Phys. Lett. 97, 171907 (2010); 10.1063/1.3506507

Direct measurement of polarization resolved transition dipole moment in InGaAs/GaAs quantum dots Appl. Phys. Lett. 82, 4552 (2003); 10.1063/1.1584514

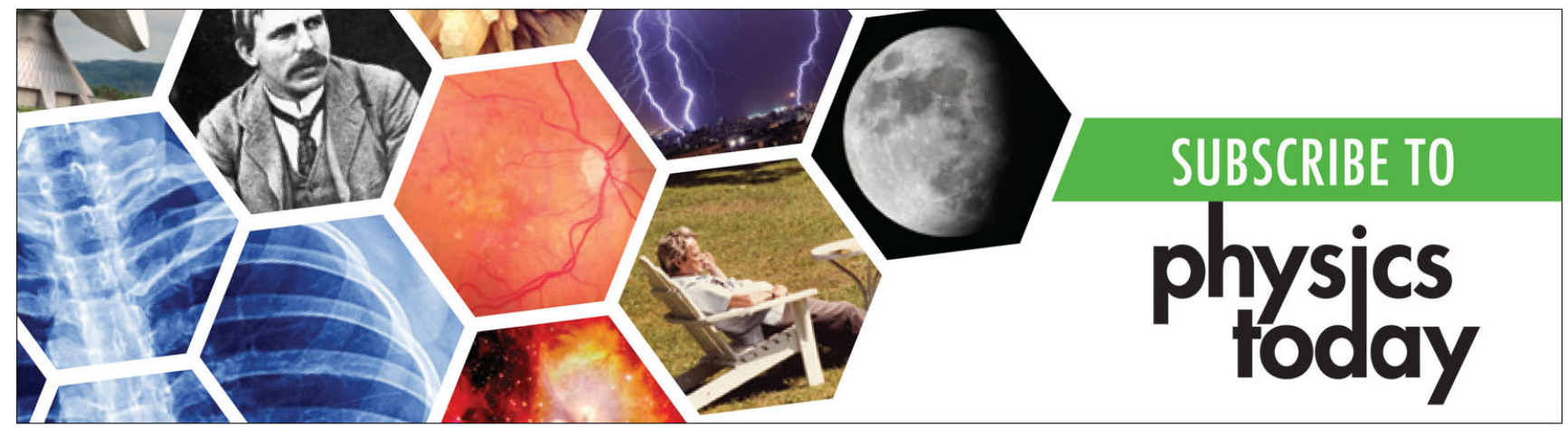




\title{
Anomalous spectral dependence of optical polarization and its impact on spin detection in InGaAs/GaAs quantum dots
}

\author{
Y. Puttisong, ${ }^{1}$ Y. Q. Huang, ${ }^{1}$ I. A. Buyanova, ${ }^{1}$ X. J. Yang,${ }^{2}$ A. Subagyo, ${ }^{2}$ K. Sueoka,${ }^{2}$ \\ A. Murayama, ${ }^{2}$ and W. M. Chen ${ }^{1}$ \\ ${ }^{1}$ Department of Physics, Chemistry and Biology, Linköping University, S-581 83 Linköping, Sweden \\ ${ }^{2}$ Graduate School of Information Science and Technology, Hokkaido University, Kita 14, Nishi 9, Kita-ku, \\ Sapporo 060-0814, Japan
}

(Received 3 September 2014; accepted 22 September 2014; published online 1 October 2014)

\begin{abstract}
We show that circularly polarized emission light from InGaAs/GaAs quantum dot (QD) ensembles under optical spin injection from an adjacent GaAs layer can switch its helicity depending on emission wavelengths and optical excitation density. We attribute this anomalous behavior to simultaneous contributions from both positive and negative trions and a lower number of photo-excited holes than electrons being injected into the QDs due to trapping of holes at ionized acceptors and a lower hole mobility. Our results call for caution in reading out electron spin polarization by optical polarization of the QD ensembles and also provide a guideline in improving efficiency of spin light emitting devices that utilize QDs. ( 2014 AIP Publishing LLC.

[http://dx.doi.org/10.1063/1.4897306]
\end{abstract}

OD systems such as semiconductor quantum dots (QDs) are known to possess a long electron spin lifetime as major events of spin relaxation promoted by carrier motion are strongly suppressed. It has been demonstrated that a spin relaxation time of electrons residing in the QD ground state can be in the range of $\mu \mathrm{s}-\mathrm{ms} .{ }^{1}$ Utilizing electron spins in QDs thus offers promising opportunities for novel devices such as spin-light-emitting diodes (spin-LEDs), spin-lasers, and spin qubits in a solid state environment. ${ }^{2-4}$ Furthermore, one of the great advantages of semiconductor QDs is their ability to encode spin information of electrons into circular polarization of emitting light and vice versa; thanks to their high radiative-recombination efficiency and a unique correlation between spin and optical polarization that is governed by the optical selection rules. ${ }^{5,6}$ This not only provides an experimental accessibility to electron spins but also enables transfer of spin information over a long distance via polarized light. From a technological point of view, vertical stacks of self-assembled $\operatorname{In}(\mathrm{Ga}) \mathrm{As}$ QDs grown on a GaAs substrate could be realized in a wafer size by employing conventional growth techniques, opening the door to fabricate spin devices in a large-scale production.

In spin light-emitting structures, spin-polarized electrons are injected into QDs from a spin source. This can be done by electrical spin injection from an adjacent ferromagnetic layer, ${ }^{7}$ a diluted magnetic semiconductor, ${ }^{8}$ Mn-doped InAs QD nanomagnets, ${ }^{9}$ or by optical spin injection through circularly polarized optical excitation of an adjacent nonmagnetic semiconductor layer under optical orientation conditions. ${ }^{6}$ Though properties of spin injectors and spin transport have received great attention during the past decade ${ }^{7-13}$ detailed studies of influence of non-resonant spin (carrier) injection density on optical polarization properties of QDs remain few. In optical orientation experiments of GaAs barriers and $\operatorname{In}(\mathrm{Ga})$ As wetting layers (WL) with a photon energy below the band-to-band (BB) optical transition energy from the spin-orbit split-off state of valence band
(VB) to conduction band (CB), $\sigma^{+}$-polarized excitation light preferably generates spin-down $\mathrm{CB}$ electrons whereas $\sigma^{-}$polarized excitation light does the opposite. If the spin orientation is preserved during spin injection, a subsequent optical transition of a positive trion $\mathrm{X}^{+}$in a positively charged QD should emit light that is co-polarized with the excitation light. Excitonic recombination in a neutral QD (namely, $\mathrm{X}^{0}$ ) is, on the other hand, expected to yield an unpolarized emission in zero magnetic field due to strong spin mixing caused by an anisotropic exchange interaction (AEI). ${ }^{14,15}$ This AEI can be suppressed to a certain extent by an Overhausser field as a result of dynamic nuclear spin polarization at high excitation power, leading to the emission that is co-polarized with the excitation light. For a negatively charged QD, recombination between one of the two spin-paired electrons and a spin-depolarized hole ${ }^{16}$ in a negative trion $\mathrm{X}^{-}$should, in principle, result in an unpolarized emission. Surprisingly, it was recently discovered that $\mathrm{X}^{-}$can in fact be counterpolarized due to spin-blockade in occupying the ground state of QDs by two electrons of the same spin orientation or due to accumulation of dark excitons in an adjacent spin injection layer. ${ }^{17-20}$ Due to possible co-existence of QDs in different charge states, it becomes not as straightforward as it seems in reading out spin information from optical polarization of a QD ensemble where emissions of various origins strongly overlap and cannot be spectrally resolved. To achieve reliable optical spin detection and efficient spin light-emitting devices, it is therefore important to understand and control key processes that determine optical polarization under various conditions of spin injection.

In this work, we examine effect of spin (carrier) injection density on spectral dependence of optical polarization from InGaAs/GaAs QD ensembles by employing the photoluminescence (PL) spectroscopy under optical spin injection from GaAs barrier layers and InGaAs WL. We shall show that nominally undoped QDs exhibit a strong variation in circular polarization of light emissions, which critically 
depends on detection energy as well as excitation photon energy and density. We propose a simple model that can provide a satisfactory explanation for all major aspects of our experimental observations.

The studied QD structures consist of three periods of Stanski-Krastanov (SK) grown $\mathrm{In}_{0.5} \mathrm{Ga}_{0.5}$ As QDs with 20$\mathrm{nm}$ thick GaAs spacers. They were grown by molecular beam epitaxy (MBE) on a semi-insulating (001) GaAs substrate with a 350-nm GaAs buffer layer. On top of most structures, another layer of QDs was grown for atomic force microscopy (AFM) studies. Fig. 1(a) shows a representative AFM image of the investigated QDs. A typical size of the QDs is $\sim 30 \mathrm{~nm}$ in diameter and $\sim 7 \mathrm{~nm}$ in height, with a density of about $3 \times 10^{10} \mathrm{~cm}^{-2}$. The structures are nominally undoped, though residual carbon acceptors $(\mathrm{C})$ were found to be present in the GaAs layers as evident from the observation of the C-related free-to-bound PL emission. PL measurements were performed at $4-10 \mathrm{~K}$ in a back-scattering geometry, in which both excitation and emission light propagated along the direction normal to the sample surface. Carrier and spin injection from the GaAs layers (or the WL) were accomplished by circularly polarized optical excitation from a continuous-wave Ti:sapphire laser with tunable wavelengths. Degrees of circular polarization of the QD PL emission, $P_{P L}$, were determined with a lock-in technique by using a photo-elastic modulator operating at $50 \mathrm{kHz}$ in conjunction with a linear polarization analyzer. $P_{P L}=\left(I^{\sigma+}-I^{\sigma-}\right) /$ $\left(I^{\sigma+}+I^{\sigma-}\right)$ where $I^{\sigma \pm}$ is PL intensity with $\sigma^{+}$and $\sigma^{-}$polarization, respectively.

A typical PL spectrum from the studied QD structures is displayed in Fig. 1(b). The PL emission originates from the ground states of excitons in the QDs that are comprised of exchange-coupled electrons and heavy-holes. The OD character of the QD light emitters was confirmed by the

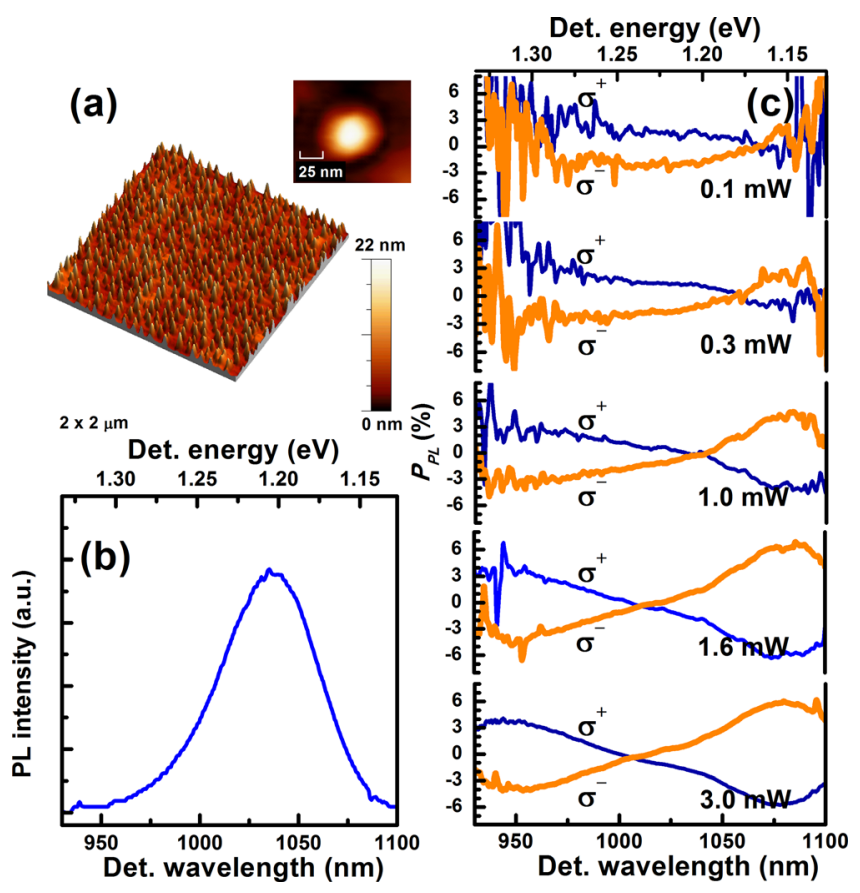

FIG. 1. (a) AFM image of the studied $\operatorname{In}_{0.5} \mathrm{Ga}_{0.5} \mathrm{As} / \mathrm{GaAs}$ QDs. (b) PL spectrum from the QD ensemble at $10 \mathrm{~K}$. (c) PL polarization spectra of the QDs at $10 \mathrm{~K}$ as a function of optical excitation power under $\sigma^{+}$and $\sigma^{-}$excitation. observation of sharp PL lines from single QDs, which can be resolved in a micro-PL spectroscopy (not show here). The PL spectrum distributes over the wavelength range of 930-1120 nm, due to a distribution in the size of the QDs. In the present study, all measurements were done under a low excitation density, thereby avoiding a significant contribution of excited state emissions, to ensure that the studied effect is indeed a character of the ground state emission. This condition also minimizes the effect of dynamic nuclear spin polarization such that $\mathrm{X}^{0}$ primarily gives rise to an unpolarized PL emission.

Representative $P_{P L}$ spectra obtained under circularly polarized optical excitation with a photon energy of $1.55 \mathrm{eV}$ (above the bandgap energy of GaAs) are plotted in Fig. 1(c) as a function of excitation power. At a low excitation power $(<0.1 \mathrm{~mW}),\left|P_{P L}\right| \sim 2 \%$ was found over the entire spectral range, which is co-polarized with the excitation light, namely, $\sigma^{+}$and $\sigma^{-}$excitation leads to positive and negative $P_{P L}$, respectively. The co-polarization behavior provides a proof for a dominant contribution of positive trions $\mathrm{X}^{+}$in the PL emissions over the entire spectral range. Interestingly, upon increasing optical excitation power, a complex pattern emerges in $P_{P L}$ spectra with the following two distinct features. First, a counter-polarized $P_{P L}$ component appears at low emission energies and is subsequently built up in strength with increasing excitation power. Second, the zero-polarization crossing point (ZCP), where $P_{P L}$ approaches zero under both $\sigma^{+}$and $\sigma^{-}$excitation, continuously shifts toward higher emission energies. The observation of the counter-polarization component in the $P_{P L}$ spectra signifies the presence of $\mathrm{X}^{-}$ (Refs. 17-20) over the corresponding spectral range. This is rather unexpected in our QD samples that are unintentionally p-type doped, as counter-polarized $P_{P L}$ was usually observed in n-type doped QD structures or in QDs where the negative charge state could be controlled by electrical bias. It is also unusual that $\mathrm{X}^{-}$seems to expand its spectral range toward higher energies or $\mathrm{X}^{+}$appears to reduce its spectral coverage by confining within the highest emission energies.

Though contributions from both $\mathrm{X}^{+}$and $\mathrm{X}^{-}$in the $\mathrm{PL}$ emission of a QD ensemble are not surprising due to a random distribution of differently charged QDs and also a random fluctuation of charge states even within a single QD, they are commonly thought to lead to a compensation in optical polarization throughout the spectral range of the QD emissions such that the total $P_{P L}$ is lower but with the same sign determined by the dominant contribution. It is particularly surprising that the spectral ranges of $\mathrm{X}^{+}$and $\mathrm{X}^{-}$seemingly vary strongly with excitation density. In other words, a strong variation in the spectral dependence of $P_{P L}$ is unexpected and so far unexplained. Here, we propose a simple model that can account for all of our experimental observations. In this model, we assume that (a) both $\mathrm{X}^{+}$and $\mathrm{X}^{-} \mathrm{PL}$ emission bands of the QD ensemble have a similar spectral lineshape that reflects the statistical distribution of the QDs in sizes; (b) these two PL emission bands are displaced by a small energy (a few meV) equivalent to the difference in the binding energies of $\mathrm{X}^{+}$and $\mathrm{X}^{-} \cdot{ }^{19,21}$ The total PL emissions are a sum of these two PL bands, ${ }^{22}$ as shown in Fig. 2(a). Within this model, a $P_{P L}$ spectrum of the QD ensemble can be simulated by 

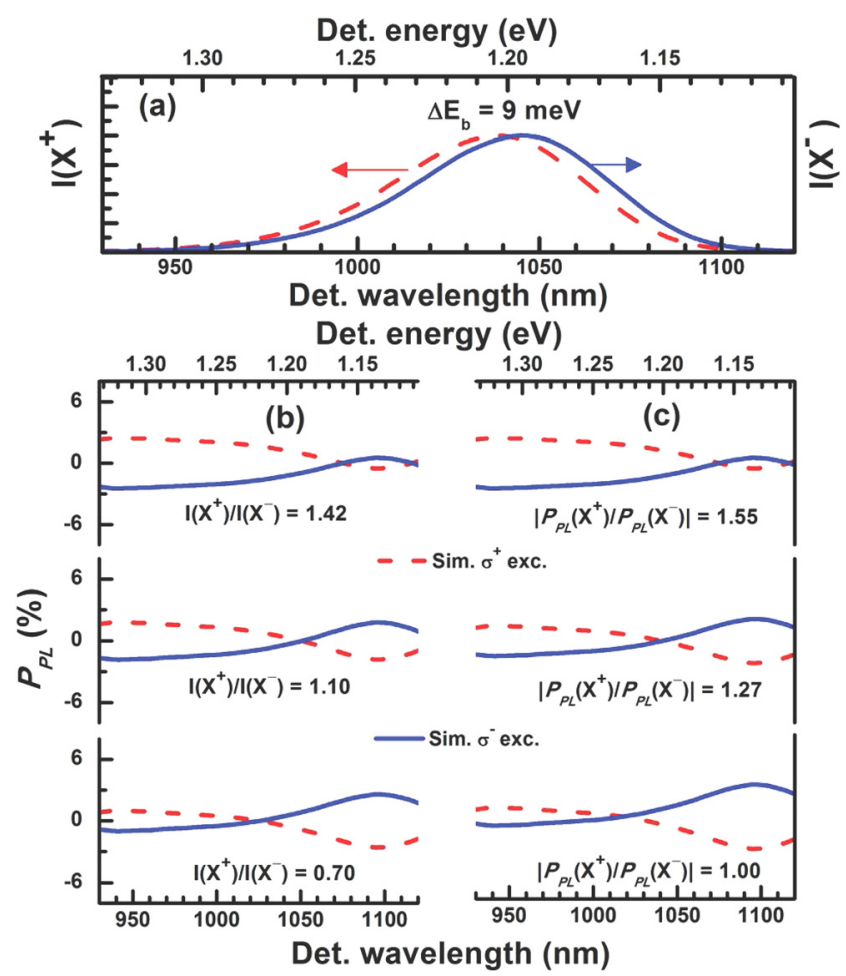

FIG. 2. (a) A model for the PL emissions from a QD ensemble by assuming overlapping of the $\mathrm{X}^{+}$and $\mathrm{X}^{-}$PL bands of the same lineshape. (b) Simulated optical polarization spectra of the QD ensemble assuming a constant $P_{P L}$ value for both $\mathrm{X}^{+}$and $\mathrm{X}^{-}$with $P_{P L}\left(\mathrm{X}^{ \pm}\right)= \pm 7 \%$, but with a varying ratio of the $\mathrm{X}^{+}$and $\mathrm{X}^{-} \mathrm{PL}$ intensity, $I\left(\mathrm{X}^{+}\right) / I\left(\mathrm{X}^{-}\right)$. (c) Simulated optical polarization spectra of the $\mathrm{QD}$ ensemble assuming a constant PL intensity for both $\mathrm{X}^{+}$and $\mathrm{X}^{-}$(taking $I\left(\mathrm{X}^{+}\right) / I\left(\mathrm{X}^{-}\right)=1$ here as an example), but with a varying value of $\left|P_{P L}\left(\mathrm{X}^{+}\right) / P_{P L}\left(\mathrm{X}^{-}\right)\right|$keeping $P_{P L}\left(\mathrm{X}^{+}\right)=+7 \%$ and a negative sign of $P_{P L}\left(\mathrm{X}^{-}\right)$.

$$
P_{P L}=\frac{P_{P L}\left(\mathrm{X}^{+}\right) I\left(\mathrm{X}^{+}\right)+P_{P L}\left(\mathrm{X}^{-}\right) I\left(\mathrm{X}^{-}\right)}{\left(I\left(\mathrm{X}^{+}\right)+I\left(\mathrm{X}^{-}\right)\right)},
$$

where $I\left(\mathrm{X}^{ \pm}\right)$and $P_{P L}\left(\mathrm{X}^{ \pm}\right)$are the intensity and optical polarization degree of the $\mathrm{X}^{ \pm}$PL band. As it can be clearly seen from Figs. 2(b) and 2(c), a change in the ratio of either PL intensity $I\left(\mathrm{X}^{+}\right) / I\left(\mathrm{X}^{-}\right)$or PL polarization $P_{P L}\left(\mathrm{X}^{+}\right) / P_{P L}\left(\mathrm{X}^{-}\right)$ can lead to a strong spectral variation in $P_{P L}$ and $\mathrm{ZCP}$ point that closely resemble our experimental results under different excitation densities as shown in Fig. 1(c).

Earlier single QD spectroscopy of GaAs/AlGaAs QDs showed that the counter-polarized $P_{P L}$ of $\mathrm{X}^{-}$can be built-up with increasing optical excitation power, ${ }^{21}$ as a result of dynamic spin polarization of the electrons such that the formation of the ground state $\mathrm{X}^{-}$with two spin-paired off electrons requires an electron and hole spin flip-flop. This could provide a possible explanation for a decreased ratio of $P_{P L}\left(\mathrm{X}^{+}\right) / P_{P L}\left(\mathrm{X}^{-}\right)$at high excitation densities, provided that $\mathrm{X}^{+}$and $\mathrm{X}^{-}$readily co-exist. On the other hand, a decrease in the PL intensity ratio of $I\left(\mathrm{X}^{+}\right) / I\left(\mathrm{X}^{-}\right)$with increasing excitation density could stem from more efficient injection of electrons than holes from the adjacent GaAs layers into the QDs due to trapping and a lower mobility of holes.

To shed light on the significance of unbalanced carrier transfer from GaAs into the QDs, we carried out a parallel study of the spectral dependence of $P_{P L}$ under optical excitation with a photon energy below the GaAs energy gap $\left(\hbar \omega_{\text {photon }}=1.39 \mathrm{eV}<E_{g}(\mathrm{GaAs})\right)$ as compared with that under excitation with $\hbar \omega_{\text {photon }}>E_{g}(\mathrm{GaAs})$. Their representative spectra are shown in Figs. 3(a) and 3(b). Unlike the case of $\hbar \omega_{\text {photon }}>E_{g}(\mathrm{GaAs})$, the counter polarization component of $P_{P L}$ diminishes when carrier injection does not involve the GaAs layer when $\hbar \omega_{\text {photon }}<E_{g}(\mathrm{GaAs})$. This is true over the entire range of excitation density as shown in Figs. 3(c) and 3(d) (For a direct comparison, we plot the absolute maximum value of counter-polarization of $P_{P L}$ $\left(\left|P_{P L}^{\text {counter }}\right|\right)$ and ZCP as a function of the total PL intensity and thus a similar exciton density under both above and below $E_{g}(\mathrm{GaAs})$ excitation conditions.). The observed absence of $\mathrm{X}^{-}$with $\hbar \omega_{\text {photon }}<E_{g}(\mathrm{GaAs})$ is in sharp contrast to a monotonous increase and a subsequent saturation of $\left|P_{P L}^{\text {counter }}\right|$ and the ZCP experimentally observed at high excitation densities when $\hbar \omega_{\text {photon }}>E_{g}(\mathrm{GaAs})$. These results show beyond doubt a direct role of carrier and spin injection from the GaAs layer.

All the experimental findings presented above can be explained if we take into account the residual p-type doping by common contaminant $\mathrm{C}$ acceptors in the GaAs layers of our nominally undoped InGaAs/GaAs QD structures and also slower trapping of holes from the GaAs layers to the QDs. In dark, the holes at the acceptors are readily transferred to the lowest-energy heavy-hole state of the QDs, leaving the QDs positively charged. Upon optical excitation when $\hbar \omega_{\text {photon }}>E_{g}(\mathrm{GaAs})$, see Fig. 4(a), photo-generated electrons in the GaAs layers have a higher probability than holes to be trapped by the QDs because holes generally have a lower mobility than electrons and additionally they can be trapped by the ionized acceptors en route to the QDs. At a low excitation density, when the number of excess electrons transferred to the QDs does not exceed that of the residual holes in the QDs, a majority of the QDs remain positively charged yielding $I\left(\mathrm{X}^{+}\right) / I\left(\mathrm{X}^{-}\right)>1$ and co-polarized PL. With increasing excitation density, however, a larger number of excess electrons are injected into the QDs such that the
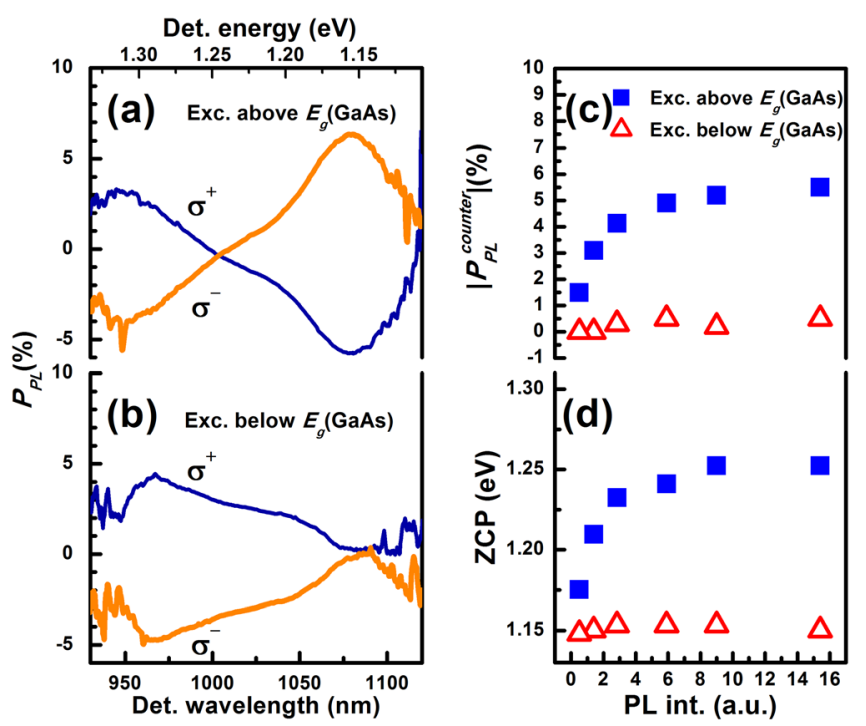

FIG. 3. (a) and (b) Polarization spectra under $\hbar \omega_{\text {photon }}>E_{g}(\mathrm{GaAs})$ and $\hbar \omega_{\text {photon }}<E_{g}(\mathrm{GaAs})$. (c) and (d) The maximum values of counter polarization and ZCP of polarization spectra as a function of ground state PL intensities (carrier densities) under the excitation above and below $E_{g}(\mathrm{GaAs})$. 
(a)

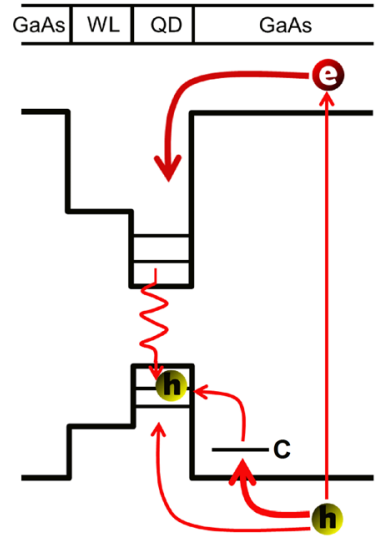

GaAs exc. (b)

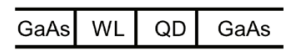

CB

VB

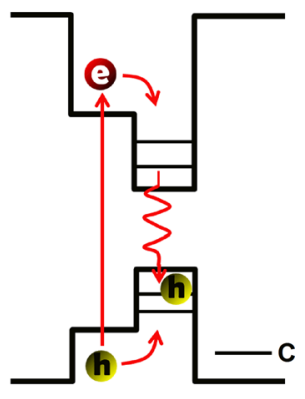

WL exc.
FIG. 4. Schematic diagram of carrier generation, trapping and recombination under optical excitation above (a) and below (b) the GaAs bandgap.

ratio of $I\left(\mathrm{X}^{+}\right) / I\left(\mathrm{X}^{-}\right)$continuously decreases and can become $I\left(\mathrm{X}^{+}\right) / I\left(\mathrm{X}^{-}\right)<1$ due to the faster trapping of electrons than holes. This trend of decrease with increasing excitation density will continue until all acceptors are neutralized and trapping of holes by acceptors ceases to occur. The minimum value of $I\left(\mathrm{X}^{+}\right) / I\left(\mathrm{X}^{-}\right)$is determined by the ratio in trapping rate into the QDs between holes and electrons. However, the situation is quite different when $\hbar \omega_{\text {photon }}<E_{g}(\mathrm{GaAs})$. In this case, see Fig. 4(b), no free carriers are generated in the GaAs layers by the optical excitation and the acceptors remain ionized regardless of excitation density used. In other words, the number of excess holes in the QDs provided by the acceptors in GaAs remains intact under the optical excitation regardless of excitation density. Even if trapping of photo-generated electrons from the WL to the QDs is faster than holes, it will require a much higher excitation density to convert positively charged QDs to negatively charged ones. Therefore, we expect that $I\left(\mathrm{X}^{+}\right) / I\left(\mathrm{X}^{-}\right)>1$ and thus the QD $\mathrm{PL}$ remains co-polarized, under the WL excitation over a similar range of photo-generation density as that when $\hbar \omega_{\text {photon }}>E_{g}(\mathrm{GaAs})$.

In conclusion, we observed an anomalous and strong spectral variation of $P_{P L}$ from nominally undoped InGaAs/ GaAs QDs. Both co- and counter-polarization PL components were observed under optical spin injection from the GaAs layers, with their intensities and spectral coverage critically depending on the emission photon energies as well as excitation density. These variations vanish when the excitation photon energy is tuned below the GaAs bandgap. We attribute the co- and counter-polarization components to the positive and negative trions, respectively. While the positively charged QDs can be accounted for by hole transfer from the unintentionally doped acceptors in the GaAs layers, an increase in the contribution of the negatively charged QDs is explained by a lower number of photo-generated holes being transferred to the QDs as compared to electrons due to their trapping by the ionized acceptors and also a lower mobility. By taking into account a small difference in the binding energies of the two exciton species, a satisfactory understanding of the observed strong spectral variations of $P_{P L}$ can be obtained. This work thus calls for cautions in directly assigning one type of optical polarization to a specific electron spin polarization in QDs. It also shows a possible pathway to overcome this ambiguity by providing holes in QDs with a density sufficient to prevent a conversion of the QDs from being positively to negatively charged within the operation range of carrier injection density employed in spin device structures. This could be done by optimizing doping level of the adjacent layers and/or exploiting gatebias-controlled hole charging devices under light illumination above the QD bandgap. ${ }^{23}$

This work was supported by Linköping University through the Professor Contracts, the Swedish Research Council (Grant No. 621-2011-4254) and the Japan Society for Promotion of Science, Grant-in-Aid for Scientific Research (S) No. 22221007.

${ }^{1}$ M. Kroutvar, Y. Ducommun, D. Heiss, M. Bichler, D. Schuh, G. Abstreiter, and J. J. Finley, Nature 432, 81 (2004).

${ }^{2}$ M. Holub and P. Bhattacharya, J. Phys. D: Appl. Phys 40, R179 (2007).

${ }^{3}$ Semiconductor Spintronics and Quantum Computation, edited by D. D. Awschalom, D. Loss, and N. Samarth (Springer, 2002).

${ }^{4}$ D. Loss and D. P. DiVincenzo, Phys. Rev. A 57, 120 (1998).

${ }^{5}$ Optical Orientation, edited by F. Meier and B. P. Zakharchenya (NorthHolland, Amsterdam, 1984).

${ }^{6}$ Spin Physics in Semiconductors, edited by M. I. Dyakonov (Springer, Berlin Heidelberg, 2008).

${ }^{7}$ G. Itshos, E. Harbord, S. K. Clowes, E. Clarke, L. F. Cohen, R. Murray, P. Van Dorpe, and W. Van Roy, Appl. Phys. Lett. 88, 022133 (2006).

${ }^{8}$ Y. Chye, M. E. White, E. Johnston-Halperin, B. D. Gerardot, D. D. Awschalom, and P. M. Petroff, Phys. Rev. B 66, 201301 (2002).

${ }^{9}$ S. Chakrabarti, M. A. Holub, P. Bhattacharya, T. D. Mishima, M. B. Samtos, M. B. Johnson, and D. A. Blom, Nano Lett. 5, 209 (2005).

${ }^{10}$ J. Beyer, I. A. Buyanova, S. Suraprapapich, C. W. Tu, and W. M. Chen, Nanotechnology 20, 375401 (2009).

${ }^{11}$ J. Beyer, P. H. Wang, I. A. Buyanova, S. Suraprapapich, C. W. Tu, and W. M. Chen, J. Phys.: Condens. Matter 24, 145304 (2012).

${ }^{12}$ A. Murayama, T. Furuta, K. Hyomi, I. Souma, Y. Oka, D. Dagnelund, I. A. Buyanova, and W. M. Chen, Phys. Rev. B 75, 195308 (2007).

${ }^{13}$ D. Dagnelund, I. A. Buyanova, W. M. Chen, A. Murayama, T. Furuta, K. Hyomi, I. Souma, and Y. Oka, Phys. Rev. B 77, 035437 (2008).

${ }^{14}$ M. Bayer, A. Kuther, A. Forchel, A. Gorbunov, V. B. Timofeev, F. Schäfer, J. P. Reithmaier, T. L. Reinecke, and S. N. Walck, Phys. Rev. Lett. 82, 1748 (1999).

${ }^{15}$ M. Paillard, X. Marie, P. Renucci, T. Amand, A. Jbrti, and J.-M. Gerard, Phys. Rev. Lett. 86, 1634 (2001).

${ }^{16}$ T. C. Damen, L. Via, J. E. Cunningham, J. Shah, and L. J. Sham, Phys. Rev. Lett. 67, 3432 (1991).

${ }^{17}$ S. Cortez, O. Krebs, S. Laurent, M. Senes, X. Marie, P. Voisin, R. Ferreira, G. Bastard, J.-M. Gerard, and T. Amand, Phys. Rev. Lett. 89, 207401 (2002).

${ }^{18}$ S. Laurent, M. Senes, O. Krebs, V. K. Kalevich, B. Urbaszek, X. Marie, T. Amand, and P. Voisin, Phys. Rev. B 73, 235302 (2006).

${ }^{19}$ A. S. Bracker, E. A. Stinaff, D. Gammon, M. E. Ware, J. G. Tischler, Al. L. Efros, D. Park, D. Gershoni, V. L. Korenev, and I. A. Merkulov, Phys. Rev. Lett. 94, 047402 (2005).

${ }^{20}$ A. Shabaev, E. A. Stinaff, A. S. Bracker, D. Gammon, A1. L. Efros, V. L. Korenev, and I. A. Merkulov, Phys. Rev. B 79, 035322 (2009).

${ }^{21}$ M. E. Ware, E. A. Stinaff, D. Gammon, M. F. Doty, A. S. Bracker, D. Gershoni, V. L. Korenev, S. C. Badescu, Y. Lyanda-Geller, and T. L. Reinecke, Phys. Rev. Lett. 95, 177403 (2005).

${ }^{22}$ For the sake of simplicity, we neglect the contributions of $\mathrm{X}^{0}$ and other exciton species in the modeling. Though this omission could lead to an overestimation of the absolute values of $P_{P L}$ as $\mathrm{X}^{0}$ gives rise to an unpolarized PL emission band that strongly overlaps with $\mathrm{X}^{+}$and $\mathrm{X}^{-}$, it will not alter the overall physical principle of the model.

${ }^{23}$ P. A. Labud, A. Ludwig, A. D. Wieck, G. Bester, and D. Reuter, Phys. Rev. Lett. 112, 046803 (2014). 